\title{
Asthma-related emergency department use: current perspectives
}

This article was published in the following Dove Press journal:

Open Access Emergency Medicine

13 July 2016

Number of times this article has been viewed

\author{
Laurie H Johnson' \\ Patricia Chambers' \\ Judith W Dexheimer ${ }^{1,2}$ \\ 'Division of Emergency Medicine, \\ ${ }^{2}$ Division of Biomedical Informatics, \\ Cincinnati Children's Hospital Medical \\ Center, Cincinnati, OH, USA
}

\begin{abstract}
Asthma is one of the most common chronic pediatric diseases. Patients with asthma often present to the emergency department for treatment for acute exacerbations. These patients may not have a primary care physician or primary care home, and thus are seeking care in the emergency department. Asthma care in the emergency department is multifaceted to treat asthma patients appropriately and provide quality care. National and international guidelines exist to help drive clinical care. Electronic and paper-based tools exist for both physicians and patients to help improve emergency, home, and preventive care. Treatment of patients with asthma should include the acute exacerbation, long-term management of controller medications, and controlling triggers in the home environment. We will address the current state of asthma research in emergency medicine in the US, and discuss some of the resources being used to help provide a medical home and improve care for patients who suffer from acute asthma exacerbations.
\end{abstract}

Keywords: asthma, pediatric, emergency department

\section{Pediatric emergency medicine}

Emergency departments (EDs) care for critically and acutely ill patients. Pediatric emergency medicine specializes in the care of infants, children, and teenagers. From 1996 to 2006, the number of ED visits increased by 3\% per year. ${ }^{1}$ During this period, the overall population-based ED-utilization rate increased by $18 \%{ }^{1}$ Pediatric visits to EDs comprise approximately $25 \%$ of all emergency visits, ${ }^{2}$ and range in acuity from life-threatening illnesses and injuries to urgent medical issues and injuries to minor acute or nonacute medical complaints. Nonemergent cases comprise $30 \%$ of pediatric emergency visits, and can be defined as "those in which immediate medical care would not be required within 12 hours" based on demographics, presenting complaints, symptoms, vital signs, and past medical history. ${ }^{3}$ Patient caregivers' perspectives on reasons for nonemergent ED visits include lack of access to physicians during nonstandard working hours and lack of health insurance, while primary care physician and ED-personnel perspectives for those visits pointed to lack of knowledge of what a "true emergency" is. ${ }^{4}$

\section{Asthma overview}

Asthma is the most common chronic childhood disease, affecting 9 million children $(12.5 \%)$ under 18 years of age in the $\mathrm{US}^{5,6}$ and placing a significant burden on the health care system. In the US, asthma is the third-leading cause of hospitalizations 
among patients less than 18 years of age. ${ }^{7}$ Nath and $\mathrm{Hsia}^{8}$ provided evidence that although the ED-visit rate for asthma increased from 2001 to 2010, this was not of statistical significance; they also noted fluctuations in asthma-visit rates among the years studied, with no pattern revealed upon regression analysis. Childhood asthma prevalence has remained relatively stable during this same time frame, with the biggest determinants of ED visits being access to care, disease severity, and socioeconomic factors. ${ }^{9,10}$ Acute asthma exacerbations require the patient to seek immediate care, and can lead to ED encounters and hospitalizations. Approximately 4 million children experience an asthma exacerbation annually, accounting for an estimated 14 million missed school days ${ }^{6}$ and more than 1.8 million ED visits each year. ${ }^{11}$ These encounters accrue more than $60 \%$ of asthmarelated costs. ${ }^{12}$ An evaluation of state Medicaid programs in 2010 estimated a combined $\$ 272$ million spent on pediatric asthma-related ED visits. ${ }^{13}$

\section{Disease burden}

The ED serves as an important access point for patients experiencing asthma exacerbations. The ED visit may be the only source for care for many asthma patients, due to a lack of a medical home or lack of a primary care provider, thus making the interaction during the ED visit even more crucial. ED asthma visits have continued to increase at a rate of approximately $13 \%$, with identification of "potentially preventable" visits. ${ }^{8}$

ED revisits after acute asthma ED visits have been studied in the short term and the long term, with revisit rates varying from $4 \%$ to $10 \%$ within the first 2 weeks following the initial ED visit. ${ }^{14,15}$ In a large multicenter study evaluating high-frequency and recurrent visits to pediatric EDs within a period of 12 months, asthma was the most common diagnosis in the first, second, and third diagnosis groups within all recurrent-visit groups (zero to four visits), as well as the most common diagnosis for high-frequency users (four or more recurrent visits). ${ }^{16}$ Those patients with one asthma-related ED visit are more likely to experience a subsequent ED visit within 1 year. ${ }^{15,17,18}$ Factors associated with an ED revisit within 1 week include age (less than 2 years), race, ethnicity, categorization of persistent asthma, government-assisted insurance, poor quality of life due to asthma symptoms, and increased use of health care resources in the preceding 12 months for asthma. ${ }^{15}$ Factors associated with an asthma-related ED visit after a hospitalization for asthma include race, governmentassisted insurance, poverty, and prior asthma-related ED hospitalization. ${ }^{18}$ Studies have concluded that increased efforts that should be implemented during the current ED visit to help prevent future ED visits include providing education, determining the level of asthma control and presence of inhaled corticosteroid (ICS) prescriptions, and ensuring scheduled follow-up. ${ }^{14,19-21}$

\section{ED treatment}

Urgent asthma care requires early identification and classification of the exacerbation severity and the prompt involvement of a coordinated provider team. Early identification, accurate assessment of the severity of airway obstruction, and response to therapy are fundamental to the ED treatment of patients experiencing acute asthma symptoms. Patients are given an initial asthma-severity rating using an asthma-scoring metric, ${ }^{22}$ peak-flow measurements, or oxygen-saturation reading with frequent reevaluation. After each assessment, care decisions should be adjusted to the new severity level, leading to a disposition decision.

Standardized care can be challenging to provide in a busy environment like the ED. Several asthma guidelines exist to help support clinicians in providing appropriate treatment, including the guidelines from the National Heart, Lung, and Blood Institute (NHLBI). ${ }^{23}$ The guidelines are general rules concerning medications and treatments clinicians should follow for optimal care. Institutions also frequently adapt and refine recommended national pathways to local care practices. Within the accepted guidelines, there is no precise definition of "acute exacerbation", although there is consensus on relieving and subsequently preventing symptoms (coughing, wheezing, chest tightness, shortness of breath) that may or may not be evidenced by measurements of lung function depending on the patient's age and severity of the exacerbation. ${ }^{24-26}$ Initial treatment for progression of asthma symptoms consists of a single short-acting $\beta$-agonist (SABA) inhalation treatment for those patients who are experiencing a mild exacerbation. Additional inhaled SABA, inhaled ipratropium bromide, and systemic corticosteroids are administered for moderate-to-severe exacerbations. ${ }^{26}$ Patients who require multiple $\beta$-agonist treatments and corticosteroids are then observed for a period of time after initial treatment to determine if further treatment is needed in the ED or as an inpatient, or if the patient can be safely discharged home. ${ }^{26,27}$ Medications that also may be initiated in the ED as second-line therapy include continuous SABA nebulization ${ }^{28}$ and a dose of intravenous magnesium sulfate. ${ }^{29,30}$ Additional therapy that is administered for those patients refractory to first- and second-line therapies or 
who present with a life-threatening exacerbation includes intravenous terbutaline, intravenous ketamine, intramuscular epinephrine, Heliox, and noninvasive positive pressure ventilation. $^{27,31-37}$

\section{ED guidelines}

Asthma-guideline utilization and adherence improves patients' clinical care. ${ }^{38,39}$ Despite the wide dissemination of guidelines and scoring systems, practice variation continues to affect treatment decisions for asthma patients. ${ }^{40,41}$ The NHLBI emergency-exacerbation management guidelines direct treatment through peak-flow readings, lung-function testing, and oxygen saturation. ${ }^{26}$ They include an asthma flow diagram that provides general principles and requires local customization to account for individual ED variations, including medications and scoring. The NHLBI guidelines emphasize early recognition of asthma symptoms and treatment stratified by acute severity. ${ }^{23}$

The temporal nature of asthma guidelines makes the more challenge to follow. A computerized system approach could help alleviate some of these challenges. These guidelines also recommend the provision of an asthma action plan (AAP) and consideration of the addition of an ICS prescription for those asthmatics discharged home after acute treatment in the ED. ${ }^{26}$

\section{ED discharge}

Patients who are discharged home from the ED require asthma-specific discharge instructions that specify which medications to continue, which new medications to begin, and when to follow-up with the primary care provider. A conundrum for many ED physicians is the question of whether to start ICS in discharged patients, as the guidelines recommend consideration of the addition of an ICS for those treated for an acute exacerbation. ${ }^{26}$ National guidelines recommend stepwise treatment based on categorization of asthma-control level, with careful consideration of patient age, impairment, and risk. ${ }^{26}$ The guidelines also advocate for provision of a written AAP (WAAP), including a strong recommendation for the addition of an ICS prescription for children discharged home after acute treatment in the ED. ${ }^{26}$

As many as $67 \%$ of pediatric ED asthma patients treated for an exacerbation were classified with persistent asthma, and less than $10 \%$ were prescribed an ICS after the ED visit. ${ }^{15,17,42,43}$ The benefits of ICS treatment of asthma include decreasing the risk of death from asthma, ${ }^{6,44}$ as well as $45 \%$ decreased ED relapse visits for asthma when compared to nonusers of ICS. ${ }^{45}$ Provider adherence to the NHLBI guidelines in an urban pediatric clinic setting of more than 3,500 patients demonstrated decreased hospitalization rates and ED visits for asthma. ${ }^{46}$ Although the majority of ED physicians agreed that patients with the categorization of persistent asthma should be treated with controller medications, ${ }^{47} \mathrm{ED}$ visits do not normally result in alteration of prescriptions for subacute or chronic asthma management. Instead, ED providers typically rely on the patient to followup with their primary care physician or subspecialist $\mathrm{t}^{42,43,48,49}$ for such management. The majority of pediatricians (80\%) support the initiation of controller medications during the ED visit, with advantages of providing controller treatment for those who are "lost to follow-up" and enforcing the importance of adherence to controller medications; concerns cited included lack of time for education in the ED setting, difficulty selecting the appropriate controller therapy based on asthma-control level, the possibility of reinforcement of ED utilization in lieu of the primary care office, inadequate communication with the primary care physician regarding the medication, and discharge plan. ${ }^{50}$ Another issue that limits the provision of ICS is the impact of managed care plans requiring prior authorization for ICS agents. This is a significant factor in patients not receiving appropriate management, because the ED physician does not have the time or the necessary continuity-care documentation necessary to obtain prior authorization for the patient to receive appropriate management.

Providing evidence-based asthma-discharge care in the ED involves categorizing the patient's current asthmacontrol level and prescribing appropriate treatment based on the specific control-level categorization determined at that visit. This is a complex, dynamic, and multistep task to perform in a busy ED setting, as it must be based on recent symptoms, past exacerbations, including emergency visits and hospitalizations, and concomitant medications. A systematic review of randomized controlled trials evaluating the efficacy of a WAAP versus none found the use of a symptom-based plan significantly reduced pediatric asthma acute care visits. ${ }^{45}$ The provision of a WAAP after ED visits for asthma was found to significantly increase patient adherence to prescribed acute and controller medications, as well as asthma control. ${ }^{51}$ However, a retrospective review of children presenting to an urban pediatric ED found that a WAAP was not prepared in $80 \%$ of visits. ${ }^{49}$ The provision of an electronically generated, personalized WAAP provides the patient and family with knowledge about their current asthma care, and allows ED physicians to provide primary care physicians and subspecialists with reliable, prompt 
communication of discharge recommendations from the visit for those patients who follow-up. The need for an ED-generated WAAP is evidenced in the variable rates of $7 \%-44 \% \%^{52-56}$ for pediatric asthma follow-up after ED visits.

\section{Follow-up and medical home}

Frequently, asthmatic children may also have inadequate access to both controller and rescue medications, due to expired prescriptions, missing or broken equipment, and lack of medications in the multiple environments in which they spend time, including alternate caregivers, school, and after-school programs. Patients with asthma may see multiple health care providers, and as a result they may be unsure of their treatment regimen and AAP. Patients may also continue to seek asthma care in an ED or urgent care setting, where there is a lack of continuity with only episodic care available, resulting in conflicting care plans depending on the provider's knowledge of pediatric asthma care. More importantly, they are left with little to no longitudinal care plan, fragmented care, and a fragmented record of care. Access to a consistent primary care provider for asthma care has been associated with increased controller-prescription fill rates, as well as reduced ED visits for asthma. ${ }^{57}$ Readmission risk for asthma was increased in those within a cohort of asthmatics with poor access to a medical home compared to those with ideal medical home access. ${ }^{58}$ However, in contrast to these findings, a recent study found that despite having access to follow-up in a "universal healthcare setting", only a third of patients completed a post-ED follow-up visit with no subsequent reduction in ED revisits or hospitalizations for asthma. ${ }^{59}$

Patients and families are frequently unaware of asthma triggers, the need for early intervention for respiratory symptoms, and the appropriate escalation plan for home care of asthma. As a result, children may present with more severe exacerbations, which require prolonged treatment and may result in hospitalization. Children with severe or "difficultto-treat exacerbation" requiring multiple courses of systemic corticosteroids are at risk for future severe exacerbations within 6 months. ${ }^{60}$ Variability of treatment of acute asthma often exists in the acute care setting when guidelines are not actively incorporated into the care plan. Adherence to most recent evidence-based medicine guidelines via clinical pathways results in more consistent respiratory scoring, as well as improved medication-ordering and -delivery times, with improved patient outcomes of reduced duration of symptoms, decreased hospital admissions, and no increase in return-visit rates. ${ }^{61-63}$

\section{Environmental factors}

Many children with asthma are repeatedly exposed to asthma and allergy triggers in their environments that exacerbate their asthma symptoms. This includes exposure to poor air quality (tobacco-smoke exposure), ${ }^{64}$ traffic-related air pollution, ${ }^{65}$ animal dander, dust mites, mold, and a lack of airconditioning. Many of these environmental factors, as well as the weather fluctuations of temperature and humidity, ${ }^{66}$ influence the severity and frequency of exacerbations. Indoor air quality has a significant impact on asthma symptoms. Airborne particles, mold, dust, and dander circulating in the air are common triggers for asthma patients. Some sources of indoor heat produce gases and particulate matter, which are airway irritants. Using a central air conditioner that includes a high-efficiency particulate-arresting filter attachment will help to reduce air pollutants. Preserving indoor humidity at low levels $(<50 \%)$ helps to control mold growth. Keeping the fluid reservoirs clean in any air-circulation device is essential in preventing the growth of organisms. ${ }^{67}$ The initiation and continued utilization of home-based health initiatives can help identify exposure issues and work toward improving patients' home environments. ${ }^{68,69}$

\section{Patient- and family-centered care}

Every child is different, as is each child's family constellation and home environment. These factors impact asthma disease course significantly. It is well known that such factors as exposure to allergens in the home (dust mites, pet dander, mold, and mildew) will exacerbate asthma symptoms. ${ }^{58,70}$ Exposure to other pollutants, such as environmental pollution (eg, exhaust, industrial fumes) and smoke from cigarettes or marijuana also aggravates the airways. ${ }^{71,72}$ In addition to exposure to allergens and irritants in the home, adherence to an asthma-treatment plan and follow-up recommendations will vary significantly depending on the child's caregivers and their attitudes and abilities to adhere to the recommended care plan. ${ }^{18}$ In this vein, it is vital that the home environment, family perspectives, and potential barriers to adherence are identified and addressed. ${ }^{73}$

Some parents do not perceive asthma as a chronic disease, but rather an episodic disease. The value of daily maintenance medications and vigilant attention to the home environment is diluted if a family does not have a clear vision for how this care affects the course of their child's asthma. Many children with asthma live in poverty, and thus live in conditions where mold, mildew, animal dander, and other irritants are hard to control. Partnering with families through education is essential to ensure that children with asthma 
continue their controller medications and use the AAP as intended. Simple, cost-conscious methods to control allergens in the home should be recommended over costly options. As stated before, connection with a medical home and access to medical attention for refills and sick visits are all part of a comprehensive plan to keep asthmatic children well. ${ }^{58,70,73}$

When a child with asthma presents to the ED with an exacerbation, it is important that this be recognized as an opportunity to review asthma care and treatment. In this teachable moment, a review of precursors to a severe asthma exacerbation, the triggers of a child's asthma, and home resources to try before an ED visit are essential. Providers need to ask about barriers to adherence in a nonjudgmental manner and help guardians and the patient to start thinking about better ways to manage symptoms, as part of the ED treatment. $^{59}$

Finally, when a child is noted to have persistent asthma symptoms and repeated ED visits for breathing issues, referral to an asthma/allergy specialist, such as a pulmonologist or allergy immunologist, should be arranged. Every child presenting to the ED for asthma-related issues needs a firm safety plan and follow-up to combat preventable complications. ${ }^{51,74}$

\section{Electronic health records}

The rate of adoption of electronic health records (EHRs) has increased from approximately $21 \%$ to $59 \%$ in pediatric institutions. ${ }^{75}$ Of these, $29 \%$ fulfill meaningful-use criteria, $71 \%$ use computerized provider order entry, and $56 \%$ have clinical guideline support. ${ }^{75}$ Approximately $46 \%$ of EDs have EHRs. ${ }^{76}$ Based on EHR data, computer-based decision support can be provided to caregivers to support clinical decision making and standardize patient care. Computerized decision-support systems are integrated with the EHR to guide treatment decisions and to aid the decision-making process at the point of care. Decision support can be delivered in various ways, such as suggesting medications, warning about adverse drug events, providing computerized guideline recommendations, or recommending a preventive care measure. Various techniques have been used to identify patients eligible for clinical decision-support systems. They are frequently developed using clinical data ${ }^{77-79}$ and then applied to real-time care areas. ${ }^{80}$ Identified barriers to lack of adherence to clinical practice guidelines include physician knowledge (eg, familiarity and awareness), physician attitudes (eg, lack of agreement, lack of outcome expectancy), and behaviors (eg, external barriers, such as patient factors or environmental factors). ${ }^{53}$ Health-information technology, including the use of decision-support systems, has been shown to have quality and efficiency benefits, especially in increasing adherence to guideline-based care ${ }^{81}$ The goal of clinical decision-support tools is to "ensure optimal, usable, and effective" patientspecific knowledge to providers at the point of care, in order "to improve the quality of health care services" 82

Computerized clinical decision support tools have been found to improve practitioner performance with disease management and drug prescribing, especially when prompts are automatically revealed to users compared to those in which users are required to activate the desired system. ${ }^{83}$ To date, the implementation of national asthma-guideline recommendations at ED discharge has not become standard practice, despite the proven benefits of decreased ED relapse rates, decreased ED visits, and increased adherence to asthma medications. $^{26,42,43,45,48,49,51,84}$

\section{EHR-based asthma protocols}

The advent and spread of EHRs has allowed clinical decision support to help improve practitioner performance in all areas of care. Asthma protocols and guidelines are frequently implemented in either electronic or combined electronic and paper-based formats. ${ }^{85}$ These protocols seek to improve patient outcomes, require care to be more compliant with national or international guidelines, decrease length of stay, and reduce ED revisits. Compliance with protocols improves patient care. ${ }^{39,86}$ In the ED, interventions improved revisits by as much as $46 \% .{ }^{87}$ Fully computerized management systems exist, but have not shown changes in asthma care in length of stay, time to disposition decision, or admission rates. ${ }^{74}$ Despite being designed to fit the workflow of the pediatric ED, no statistically significant changes were found. These computerized systems are well studied and perform well, ${ }^{88-90}$ but guideline adherence remains an issue.

\section{Asthma TreatSmart}

The evidence-based treatment of asthma relies on the selection of rescue (SABA) and controller medications (ICS, intranasal steroids, daily allergy medications) based on each child's specific triggers and pattern of disease exacerbation. Navigating the complex choices of medications, dosages, and routes of administration that adhere to NHLBI guidelines is daunting in the ED setting. As a result, it is quite common that ED physicians avoid prescribing controller medication and defer this to the primary care physician. ${ }^{74}$ As stated previously, many children with uncontrolled asthma have limited access to primary care or use the ED for episodic care without adequate follow-up. Many primary care physicians see children only rarely after infancy. Some primary care physicians 
may not be familiar or comfortable with the most up-to-date recommendations for treatment of persistent asthma symptoms. For these reasons, symptom capture and initiation of evidence-based treatment of chronic asthma symptoms is important during ED visits for asthma symptoms. ${ }^{51,58,59,70-72}$

Asthma TreatSmart is a stand-alone web-based system developed at Cincinnati Children's Hospital Medical Center by the Division of Pulmonary Medicine. It is based on national asthma guidelines. The application uses historic information from the EHR and parent/patient-reported symptom inventories to assign an asthma-severity classification. Patient- and parent-reported medication use is integrated into the program. The information can be collected and entered by ancillary staff (ie, respiratory therapist, nurse, medical assistant) and verified by the treating physician. The program then generates treatment recommendations, including a personalized AAP and controller-medication recommendations, that are evidence-based and easy to print and implement. It also generates recommendations for follow-up plans. In addition, the information summary and treatment recommendations can be copied and pasted back to the EHR.

Utilization of this web-based system allows the busy ED clinician to provide a comprehensive review of asthma symptoms and medication use. The program generates userfriendly, evidence-based guidelines, and is a very effective tool for ensuring children with asthma receive preventive measures for controlling asthma symptoms.

\section{Asthma Home Health Program}

As previously stated, assessment of the home milieu and patient/parent activation are vital components of the aftercare of a child with asthma. ${ }^{58,70}$ The development of an Asthma Home Health Program is aimed at taking the education, assessment of the home environment, the procurement of needed equipment and medication, and the implementation of the follow-up plan to the patient's home..$^{80,91}$

All children presenting with asthma symptoms to the ED are recommended for screening. Screening questions assess asthma severity (admission and ED-encounter data), patient-reported medication-adherence information, and provider-reported concerns about education and the home environment.

If a child screens as eligible, parents are then introduced to the program and encouraged to fill out intake forms. From there, a centralized process is in place for more in-depth screening and completion of enrollment in eligible and willing families. Enrolled families experience 3-5 in-home visits performed by a trained asthma home health nurse,
Table I Home-care nurse visits

\begin{tabular}{|c|c|}
\hline \multirow[t]{8}{*}{ Educational } & Review of anatomy, physiology, and asthma medication \\
\hline & Self-management assessment and education \\
\hline & Interactive engagement activities \\
\hline & Reinforcement of the importance of consistent \\
\hline & adherence to asthma-prevention measures and \\
\hline & identification of early asthma-exacerbation symptoms \\
\hline & and treatment \\
\hline & Proper use of mouthpiece and mask \\
\hline \multirow[t]{4}{*}{ Clinical } & Assessment of environmental triggers \\
\hline & Asthma Control Test scoring \\
\hline & Comprehensive physical assessment \\
\hline & Review of asthma action plan \\
\hline
\end{tabular}

with the goal of improved self-management of asthma and reduced symptom severity. Components of the program are described in Table 1.

Additionally, the coordination of care between the homecare authorization team, home-care asthma nurse, and primary care physician, as well as secondary physicians when requested (allergy/immunology, pulmonology), is important to maintain, to help coordinate and improve patient care. Included in this is equipment and medication management. This requires that all asthma medications and equipment are present and function for home use, including:

- assistance with establishing a medical home (primary care physician)

- referral to allergy/immunology or pulmonology as indicated

- assistance in signing up for Medicaid or insurance.

Finally, patients not at their goal may continue in the program or be re-referred at a future time.

\section{Calls to action: how can we improve?}

Asthma morbidity continues to increase in the US. Guideline and protocol implementations in the ED are improving patient care and outcomes, but a coordinated effort is needed to continue to improve patient care. There are several key things that can be done to help improve care in the ED, and one of these is the consistent use of a standardized, evidence-based classification system for asthma severity. Severity classification is key to following guideline recommendations. Coupled with this is the need for the dissemination of evidence-based guidelines for asthma care in the ED. These guidelines and protocols should address stepwise, hour-by-hour treatment algorithms, as well as contain a standardized objective respiratory scoring system and protocol for when to score and how to use the score. All of these will help to standardize asthma 
care and lead to better patient outcomes. Leveraging the EHR and associated electronic systems can help these issues.

A standard approach to asthma discharge can help improve patient-provider and provider-provider communication. Continued improvement of communication between primary care providers and ED physicians is important to help providers know which questions to ask, how to ask them, and how to interpret answers, making sure that when discharged, the patient has a WAAP in hand, educational goals set during the ED visit are met, such as how to use albuterol at home, if the patient has a clear plan for obtaining steroids, and planned and set follow-up with the patient's medical home.

Asthma continues to be an enormous medical and public health concern within the pediatric population. The majority of pediatric asthmatics are treated not in pediatric EDs but in community ED, urgent care, and primary care settings; therefore, dissemination of evidence-based guidelines for acute asthma care with standardized treatment plans is essential to providing optimal care. Consistent utilization of a standardized, evidenced-based classification system within the ED workflow, to not only establish level of asthma control at each visit, but also to identify acute severity at each visit, would enhance treatment and discharge plans with the ultimate goal of improving patient outcomes. Finally, assurance of continuing patient and family education with compliance of controller and rescue medications, coupled with a reliable medical home for follow-up and continuity of care, are key elements in the struggle to manage this chronic disease.

\section{Disclosure}

The authors report no conflicts of interest in this work.

\section{References}

1. Pitts SR, Niska RW, Xu J, Burt CW. National Hospital Ambulatory Medical Care Survey: 2006 emergency department summary. Natl Health Stat Report. 2008;(7):1-38.

2. Luo X, Liu G, Frush K, Hey LA. Children's health insurance status and emergency department utilization in the United States. Pediatrics. 2003;112(2):314-319.

3. Owens PL, Zodet MW, Berdahl T, Dougherty D, McCormick MC, Simpson LA. Annual report on health care for children and youth in the United States: focus on injury-related emergency department utilization and expenditures. Ambul Pediatr. 2008;8(4):219-240.e17.

4. Ben-Isaac E, Schrager SM, Keefer M, Chen AY. National profile of nonemergent pediatric emergency department visits. Pediatrics. 2010;125(3): 454-459.

5. Centers for Disease Control and Prevention. FastStats: asthma. 2016. Available from: http://www.cdc.gov/nchs/fastats/asthma.htm. Accessed October 21, 2015.

6. Centers for Disease Control and Prevention. QuickStats: percentage of children aged $<18$ years who have ever had asthma diagnosed, by age group: United States, 2003. 2005. Available from: http://www.cdc.gov/ $\mathrm{mmwr} / \mathrm{preview} / \mathrm{mmwhhtml} / \mathrm{mm} 5416 \mathrm{a} 5 . \mathrm{htm}$. Accessed October 21, 2015.
7. Eder W, Ege MJ, von Mutius E. The asthma epidemic. N Engl J Med. 2006;355(21):2226-2235.

8. Nath JB, Hsia RY. Children's emergency department use for asthma, 2001-2010. Acad Pediatr. 2015;15(2):225-230.

9. Centers for Disease Control and Prevention. Asthma facts: CDC's National Asthma Control Program grantees. 2013. Available from: http://www.cdc.gov/asthma/pdfs/asthma_facts_program_grantees.pdf. Accessed March 24, 2016.

10. Centers for Disease Control and Prevention. Asthma. 2016. Available from: http://www.cdc.gov/asthma/default.htm. Accessed March 24, 2016.

11. Allergy and Asthma Advocate. Quarterly Patient Newsletter of the American Academy of Allergy, Asthma and Immunology. 2004. Available from: http://www.cdc.gov/nchs/data/ahcd/nhamcs_emergency/2011_ed_web_tables.pdf. Accessed June 8, 2016.

12. Lozano P, Sullivan SD, Smith DH, Weiss KB. The economic burden of asthma in US children: estimates from the National Medical Expenditure Survey. J Allergy Clin Immunol. 1999;104(5):957-963.

13. Pearson WS, Goates SA, Harrykissoon SD, Miller SA. State-based Medicaid costs for pediatric asthma emergency department visits. Prev Chronic Dis. 2014;11:E108.

14. Emerman CL, Woodruff PG, Cydulka RK, Gibbs MA, Pollack CV Jr, Camargo CA Jr. Prospective multicenter study of relapse following treatment for acute asthma among adults presenting to the emergency department. Chest. 1999;115(4):919-927.

15. Walsh-Kelly CM, Kelly KJ, Drendel AL, Grabowski L, Kuhn EM. Emergency department revisits for pediatric acute asthma exacerbations: association of factors identified in an emergency department asthma tracking system. Pediatr Emerg Care. 2008;24(8):505-510.

16. Alpern ER, Clark AE, Alessandrini EA, et al. Recurrent and highfrequency use of the emergency department by pediatric patients. Acad Emerg Med. 2014;21(4):365-373.

17. Kwok MY, Walsh-Kelly CM, Gorelick MH, Grabowski L, Kelly KJ. National Asthma Education and Prevention Program severity classification as a measure of disease burden in children with acute asthma. Pediatrics. 2006;117(4 Pt 2):S71-S77.

18. Tolomeo C, Savrin C, Heinzer M, Bazzy-Asaad A. Predictors of asthmarelated pediatric emergency department visits and hospitalizations. $J$ Asthma. 2009;46(8):829-834.

19. Guttmann A, Zagorski B, Austin PC, et al. Effectiveness of emergency department asthma management strategies on return visits in children: a population-based study. Pediatrics. 2007;120(6): e1402-e1410.

20. Stevens MW, Gorelick MH. Short-term outcomes after acute treatment of pediatric asthma. Pediatrics. 2001;107(6):1357-1362.

21. To T, Wang C, Dell S, et al. Risk factors for repeat adverse asthma events in children after visiting an emergency department. Ambul Pediatr. 2008;8(5):281-287.

22. Qureshi F, Pestian J, Davis P, Zaritsky A. Effect of nebulized ipratropium on the hospitalization rates of children with asthma. $N$ Engl J Med. 1998;339(15):1030-1035.

23. National Asthma Education and Prevention Program. Expert panel report 2: guidelines for the diagnosis and management of asthma. 1997. Available from: http://www.nhlbi.nih.gov/health-pro/guidelines/archive/ asthma-expert-panel-report. Accessed October 21, 2015.

24. Fuhlbrigge A, Peden D, Apter AJ, et al. Asthma outcomes: exacerbations. J Allergy Clin Immunol. 2012;129(3 Suppl):S34-S48.

25. Global Initiative for Asthma. Global strategy for asthma management and prevention. 2015. Available from: http://ginasthma.org/2016-ginareport-global-strategy-for-asthma-management-and-prevention/. Accessed June 8, 2016.

26. National Asthma Education and Prevention Program. Expert Panel Report 3: Guidelines for the Diagnosis and Management of Asthma. Bethesda (MD): National Heart, Lung, and Blood Institute; 2007. Available from: http://www.nhlbi.nih.gov/files/docs/guidelines/asthgdln.pdf. Accessed May 10, 2016. 
27. Kelly AM, Kerr D, Powell C. Is severity assessment after one hour of treatment better for predicting the need for admission in acute asthma? Respir Med. 2004;98(8):777-781.

28. Papo MC, Frank J, Thompson AE. A prospective, randomized study of continuous versus intermittent nebulized albuterol for severe status asthmaticus in children. Crit Care Med. 1993;21(10):1479-1486.

29. Cheuk DK, Chau TC, Lee SL. A meta-analysis on intravenous magnesium sulphate for treating acute asthma. Arch Dis Child. 2005;90(1):74-77.

30. Torres S, Sticco N, Bosch JJ, et al. Effectiveness of magnesium sulfate as initial treatment of acute severe asthma in children, conducted in a tertiary-level university hospital: a randomized, controlled trial. Arch Argent Pediatr. 2012;110(4):291-296.

31. Basnet S, Mander G, Andoh J, Klaska H, Verhulst S, Koirala J. Safety, efficacy, and tolerability of early initiation of noninvasive positive pressure ventilation in pediatric patients admitted with status asthmaticus: a pilot study. Pediatr Crit Care Med. 2012;13(4):393-398.

32. Bigham MT, Jacobs BR, Monaco MA, et al. Helium/oxygen-driven albuterol nebulization in the management of children with status asthmaticus: a randomized, placebo-controlled trial. Pediatr Crit Care Med. 2010;11(3):356-361.

33. Bogie AL, Towne D, Luckett PM, Abramo TJ, Wiebe RA. Comparison of intravenous terbutaline versus normal saline in pediatric patients on continuous high-dose nebulized albuterol for status asthmaticus. Pediatr Emerg Care. 2007;23(6):355-361.

34. Jat KR, Chawla D. Ketamine for management of acute exacerbations of asthma in children. Cochrane Database Syst Rev. 2012;11:CD009293.

35. Koninckx M, Buysse C, de Hoog M. Management of status asthmaticus in children. Paediatr Respir Rev. 2013;14(2):78-85.

36. Mitra A, Bassler D, Goodman K, Lasserson TJ, Ducharme FM. Intravenous aminophylline for acute severe asthma in children over two years receiving inhaled bronchodilators. Evidence-Based Child Health: A Cochrane Review Journal 2006;1(1):101-146.

37. Wheeler DS, Jacobs BR, Kenreigh CA, Bean JA, Hutson TK, Brilli RJ. Theophylline versus terbutaline in treating critically ill children with status asthmaticus: a prospective, randomized, controlled trial. Pediatr Crit Care Med. 2005;6(2):142-147.

38. Grimshaw JM, Eccles MP, Walker AE, Thomas RE. Changing physicians' behavior: what works and thoughts on getting more things to work. J Contin Educ Health Prof. 2002;22(4):237-243.

39. Scribano PV, Lerer T, Kennedy D, Cloutier MM. Provider adherence to a clinical practice guideline for acute asthma in a pediatric emergency department. Acad Emerg Med. 2001;8(12):1147-1152.

40. Cabana MD, Slish KK, Nan B, Lin X, Clark NM. Asking the correct questions to assess asthma symptoms. Clin Pediatr (Phila). 2005;44(4): 319-325.

41. Halterman JS, Yoos HL, Kaczorowski JM, et al. Providers underestimate symptom severity among urban children with asthma. Arch Pediatr Adolesc Med. 2002;156(2):141-146.

42. Garro AC, Asnis L, Merchant RC, McQuaid EL. Frequency of prescription of inhaled corticosteroids to children with asthma in U.S. emergency departments. Acad Emerg Med. 2011;18(7):767-770.

43. Lovinsky S, Rastogi D. Prescription habits for preventative medications among pediatric emergency department physicians at an inner-city teaching hospital. J Asthma. 2010;47(9):1011-1014.

44. Suissa S, Ernst P, Benayoun S, Baltzan M, Cai B. Low-dose inhaled corticosteroids and the prevention of death from asthma. NEngl J Med. 2000;343(5):332-336.

45. Sin DD, Man SF. Low-dose inhaled corticosteroid therapy and risk of emergency department visits for asthma. Arch Intern Med. 2002;162(14):1591-1595.

46. Cloutier MM, Hall CB, Wakefield DB, Bailit H. Use of asthma guidelines by primary care providers to reduce hospitalizations and emergency department visits in poor, minority, urban children. J Pediatr. 2005; 146(5):591-597

47. Scarfone RJ, Zorc JJ, Angsuco CJ. Emergency physicians' prescribing of asthma controller medications. Pediatrics. 2006;117(3): $821-827$.
48. Blais L, Beauchesne MF. Use of inhaled corticosteroids following discharge from an emergency department for an acute exacerbation of asthma. Thorax. 2004;59(11):943-947.

49. Ly CD, Dennehy CE. Emergency department management of pediatric asthma at a university teaching hospital. Ann Pharmacother. 2007; 41(10):1625-1631

50. Sampayo EM, McLoughlin RJ, Tsevdos D, Alam S, Zorc JJ. Pediatricians support initiation of asthma controller medications in the emergency department: a national survey. Pediatr Emerg Care. 2015;31(8): $545-550$.

51. Ducharme FM, Zemek RL, Chalut D, et al. Written action plan in pediatric emergency room improves asthma prescribing, adherence, and control. Am J Respir Crit Care Med. 2011;183(2):195-203.

52. Baren JM, Shofer FS, Ivey B, et al. A randomized, controlled trial of a simple emergency department intervention to improve the rate of primary care follow-up for patients with acute asthma exacerbations. Ann Emerg Med. 2001;38(2):115-122.

53. Cabana MD, Bruckman D, Bratton SL, Kemper AR, Clark NM. Association between outpatient follow-up and pediatric emergency department asthma visits. J Asthma. 2003;40(7):741-749.

54. Petersen DL, Murphy DE, Jaffe DM, et al. A tool to organize instructions at discharge after treatment of asthmatic children in an emergency department. J Asthma. 1999;36(7):597-603.

55. Smith SR, Jaffe DM, Fisher EB Jr, Trinkaus KM, Highstein G, Strunk $\mathrm{RC}$. Improving follow-up for children with asthma after an acute emergency department visit. J Pediatr. 2004;145(6):772-777.

56. Zorc JJ, Scarfone RJ, Li Y, et al. Scheduled follow-up after a pediatric emergency department visit for asthma: a randomized trial. Pediatrics. 2003;111(3):495-502.

57. Smith SR, Wakefield DB, Cloutier MM. Relationship between pediatric primary provider visits and acute asthma ED visits. Pediatr Pulmonol.2007; 42(11):1041-1047.

58. Auger KA, Kahn RS, Davis MM, Beck AF, Simmons JM. Medical home quality and readmission risk for children hospitalized with asthma exacerbations. Pediatrics. 2013;131(1):64-70.

59. Li P, To T, Guttmann A. Follow-up care after an emergency department visit for asthma and subsequent healthcare utilization in a universalaccess healthcare system. J Pediatr. 2012;161(2):208-213.e1.

60. Haselkorn T, Zeiger RS, Chipps BE, et al. Recent asthma exacerbations predict future exacerbations in children with severe or difficult-to-treat asthma. J Allergy Clin Immunol. 2009;124(5):921-927.

61. Bekmezian A, Fee C, Weber E. Clinical pathway improves pediatrics asthma management in the emergency department and reduces admissions. J Asthma. 2015;52(8):806-814.

62. Norton SP, Pusic MV, Taha F, Heathcote S, Carleton BC. Effect of a clinical pathway on the hospitalisation rates of children with asthma: a prospective study. Arch Dis Child. 2007;92(1):60-66.

63. Zemek R, Plint A, Osmond $\mathrm{MH}$, et al. Triage nurse initiation of corticosteroids in pediatric asthma is associated with improved emergency department efficiency. Pediatrics. 2012;129(4):671-680.

64. Kit BK, Simon AE, Brody DJ, Akinbami LJ. US prevalence and trends in tobacco smoke exposure among children and adolescents with asthma. Pediatrics. 2013;131(3):407-414.

65. Newman NC, Ryan PH, Huang B, Beck AF, Sauers HS, Kahn RS. Traffic-related air pollution and asthma hospital readmission in children: a longitudinal cohort study. J Pediatr. 2014;164(6):1396-1402.e1.

66. Mireku N, Wang Y, Ager J, Reddy RC, Baptist AP. Changes in weather and the effects on pediatric asthma exacerbations. Ann Allergy Asthma Immunol. 2009;103(3):220-224.

67. Asthma and Allergy Foundation of America. Pollen allergy: what is a pollen allergy? 2015. Available from: http://www.aafa.org/page/pollenallergy.aspx. Accessed March 1, 2016.

68. Krieger JW, Song L, Takaro TK, Stout J. Asthma and the home environment of low-income urban children: preliminary findings from the SeattleKing County healthy homes project. J Urban Health. 2000;77(1):50-67.

69. Verhoeff AP, Burge HA. Health risk assessment of fungi in home environments. Ann Allergy Asthma Immunol. 1997;78(6):544-556. 
70. Beck AF, Simmons JM, Sauers HS, et al. Connecting at-risk inpatient asthmatics to a community-based program to reduce home environmental risks: care system redesign using quality improvement methods. Hosp Pediatr. 2013;3(4):326-334.

71. Kline-Krammes S, Patel NH, Robinson S. Childhood asthma: a guide for pediatric emergency medicine providers. Emerg Med Clin North Am. 2013;31(3):705-732.

72. Patridge R, Abramo T. Acute asthma in the pediatric emergency department. 2008. Available from: https:/www.ebmedicine.net/topics php?paction=showTopic\&topic_id=159. Accessed February 11, 2016.

73. Sabaté E, editor. Adherence to Long-Term Therapies: Evidence for Action. Geneva: World Health Organization; 2013. Available from: http://www.who.int/chp/knowledge/publications/adherence_introduc tion.pdf. Accessed February 11, 2016.

74. Dexheimer JW, Abramo TJ, Arnold DH, et al. An asthma management system in a pediatric emergency department. Int J Med Inform. 2013;82(4):230-238.

75. Nakamura MM, Harper MB, Jha AK. Change in adoption of electronic health records by US children's hospitals. Pediatrics. 2013;131(5) e1563-e1575.

76. Geisler BP, Schuur JD, Pallin DJ. Estimates of electronic medical records in U.S. emergency departments. PLoS One. 2010;5(2):e9274.

77. Sanders DL, Aronsky D. Prospective evaluation of a Bayesian network for detecting asthma exacerbations in a pediatric emergency department AMIA Annu Symp Proc. 2006;2006:1085.

78. Sanders DL, Aronsky D. Detecting asthma exacerbations in a pediatric emergency department using a Bayesian network. AMIA Annu Symp Proc. 2006;2006:684-688.

79. Sanders DL, Gregg W, Aronsky D. Identifying asthma exacerbations in a pediatric emergency department: a feasibility study. Int J Med Inform. 2007;76(7):557-564

80. Dexheimer JW, Arnold DH, Johnson K, et al. Randomized controlled trial of a computerized asthma management system in a pediatric emergency department. Poster presented at: AMIA 2011 Annual Symposium; October 22-26, 2011; Washington, DC.

81. Chaudhry B, Wang J, Wu S, et al. Systematic review: impact of health information technology on quality, efficiency, and costs of medical care. Ann Intern Med. 2006;144(10):742-752.
82. Osheroff JA, Teich JM, Middleton B, Steen EB, Wright A, Detmer DE A roadmap for national action on clinical decision support. J Am Med Inform Assoc. 2007;14(2):141-145.

83. Garg AX, Adhikari NK, McDonald H, et al. Effects of computerized clinical decision support systems on practitioner performance and patient outcomes: a systematic review. JAMA. 2005;293(10):1223-1238.

84. Rowe BH, Bota GW, Fabris L, Therrien SA, Milner RA, Jacono J. Inhaled budesonide in addition to oral corticosteroids to prevent asthma relapse following discharge from the emergency department: a randomized controlled trial. JAMA. 1999;281(22):2119-2126.

85. Dexheimer JW, Borycki EM, Chiu KW, Johnson KB, Aronsky D. A systematic review of the implementation and impact of asthma protocols. BMC Med Inform Decis Mak. 2014;14:82.

86. Yasin F, Martin C, Bolger T. The compliance to acute asthma management protocols in paediatric emergency department. Ir Med J. 2013;106(10):305-307.

87. Lukacs SL, France EK, Baron AE, Crane LA. Effectiveness of an asthma management program for pediatric members of a large health maintenance organization. Arch Pediatr Adolesc Med. 2002;156(9) $872-876$.

88. Hoeksema LJ, Bazzy-Asaad A, Lomotan EA, et al. Accuracy of a computerized clinical decision-support system for asthma assessment and management. J Am Med Inform Assoc. 2011;18(3):243-250.

89. Shiffman RN. Towards effective implementation of a pediatric asthma guideline: integration of decision support and clinical workflow support. In Proceedings of the annual symposium on computer application in medical care, American Medical Informatics Association; 1994:797-801.

90. Shiffman RN, Freudigman M, Brandt CA, Liaw Y, Navedo DD. A guideline implementation system using handheld computers for office management of asthma: effects on adherence and patient outcomes. Pediatrics. 2000;105(4 Pt 1):767-773.

91. McCarthy D, Cohen A. The Cincinnati Children's Hospital Medical Center's Asthma Improvement Collaborative: enhancing quality and coordination of care. 2013. Available from: http://www.commonwealthfund.org/ /media/Files/Publications/Case\%20Study/2013/Jan/1660_ McCarthy_care_transitions_Cincinnati_case_study_v2.pdf. Accessed November 23, 2015
Open Access Emergency Medicine

\section{Publish your work in this journal}

The Open Access Emergency Medicine is an international, peerreviewed, open access journal publishing original research, reports, editorials, reviews and commentaries on all aspects of emergency medicine. The manuscript management system is completely online and includes a very quick and fair peer-review system, which is all

\section{Dovepress}

easy to use. Visit http://www.dovepress.com/testimonials.php to read real quotes from published authors. 